\title{
Concurrence of Primary Cutaneous Extra Mammary Paget's Disease and Squamous Cell Carcinoma in situ of Vulva: A Case Report
}

\author{
Fatemeh Samiee-Rad $^{1 *}$, Sohayla Farajee ${ }^{2}$
}

1. Department of Pathology, School of Medicine, Metabolic Diseases Research Center, Qazvin University of Medical Sciences, Qazvin, Iran

2. School of Medicine, Metabolic Diseases Research Center, Qazvin University of Medical Sciences, Qazvin, Iran

Scan to discover online

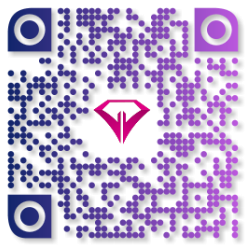

\begin{tabular}{c}
\hline Main Subjects: \\
Gynecologic Pathology \\
\hline
\end{tabular}

Received 08 Sep 2018;

Accepted 31 Oct 2019;

Published Online 26 Dec 2019;

10.30699/IJP.2019.93259.1906

PMCID: PMC6995679

PMID: 32095151

\author{
ABSTRACT
}

Extramammary Paget's disease and vulvar intraepithelial neoplasia are common lesions of vulve, but synchronous occurrence is rare in a same location. Herein we describe a concurrence of primary cutaneous extra mammary Paget's disease and squamous cell carcinoma in situ of vulva in an Iranian women. A 59 year old woman, initially presented to Kosar teaching hospital gynecology clinic April 2017 with a single, well defined, scaly, white ulcerated mass, $2 \mathrm{~cm}$ in diameter on right minor labia, but other examinations were normal. She had no urinary or gastrointestinal symptoms. Incisional biopsies from the mass represented concurrence extramammary Paget's disease and VIN3. She underwent radical vulvectomy .No evidence of disease recurrence was noted after 16 months follow up. Participant consent was obtained before patient was enrolled in this study. There are lot of diseases that involve genitalia and lead to vulvar lesions. But in this rare case, we diagnosed concurrence of extramammary Paget's disease and vulvar intraepithelial neoplasia in a same location. Additionally, the clinical presentation as a vulvar mass was found unusual. Therefore, we report the case to sensitize gynecologists and pathologists for uncommon pathologies and their manifestations in vulva.

KEYWORDS: Extramammary Paget's disease, Intraepithelial neoplasia, Vulve

Corresponding Information:

Fatemeh. Samiee-Rad, Dept. of Pathology, School of Medicine, Metabolic Diseases Research Center, Qazvin University of Medical Sciences, Qazvin, Iran Email: fsamieerad@gmail.com

Copyright $\odot$ 2020. This is an open-access article distributed under the terms of the Creative Commons Attribution- 4.0 International License which permits Share, copy and redistribution of the material in any medium or format or adapt, remix, transform, and build upon the material for any purpose, even commercially.

\section{Introduction}

Paget's disease is a rare neoplasm of the nipple (1). Extramammary Paget's disease (EMPD), which was first described by Crocker in 1888 (1), is an intraepithelial adenocarcinoma (2) in apocrine cells rich glandular regions (1). It's more common in Cacausian women and Asian men (1).

The most frequently affected areas include: vulva, scrotum, penis and perineum (3). Pruritus is the most common patient's symptom (4). Standard treatment is a surgical resection with wide safe margins (2). Squamous intraepithelial lesions were first described by Bowen in 1912, it is also named squamous cell carcinoma (SCC) in situ or Bowen disease, but some years after, this term changed to vulvar intraepithelial neoplasia (VIN) (5). Associated symptoms are pruritus, pain and skin lesion (4). Lesions can be red, white or pigmented, either smooth or ridged and present as striae or ulcers. However, it often manifests by erythematous plaques and is uncommon to involve genital area (2). Surgical treatment is an effective method in removal of premalignant lesions but in VIN, relapse is very common (2). EMPD and VIN alone are common lesions of vulve, but concurrence of these neoplasms in a same lesions, is a rare event $(1,5)$.
As mentioned before, EMPD and in situ SCC usually manifest as plaque (2), but in this case, a patient is presented with a vulvar mass, which is an uncommon presentation.

Tumoral cells of VIN3 origin from the intraepithermal portion of the hair follicle (acrotrichium) (6) or arise from multipotential cells situated along the epithermal basal layer that differentiated along squamous cells (7). On the other hand tumoral cells of EMPD derivate from the intraepithermal part of glands (acrosyrinngium) (8) or arise from multipotential cells situated in the direction of the epithermal basal layer that transform along sweat glands series (9). About the origin of two above lesions, the second interpretation being the more acceptable, explains concurrence of them in a lesion $(8,9)$.

Herein we describe a concurrence of primary cutaneous extramammary Paget's disease and squamous cell carcinoma in situ of vulva, an Iranian women in order to sensitize gynecologists and pathologists to this diagnostic challenge. 


\section{Materials and Methods}

The patient was a non-smoker 59-year-old healthy married, menopausal woman presented with 1-year history of consistent and progressive vulvar pruritus and a vulvar mass lesion to the gynecology clinic at Kosar teaching Hospital in April 2017 without any former treatment. She had good appetite, regular and painless defecation, without nausea or vomiting, hematochezia, dysuria, hematuria or other urinary signs.

She had a medical history of severe cervicitis about six years prior to her refer to hospital. Accompanied pathology report of colposcopic biopsy shown marked acute on chronic cervicitis with koilocytic changes.

There were not any expressions of significant family history of previous medical or surgical pathology in the patient.

The patient on general appearance was healthy, well hydrated and afebrile, with no signs of anemia, jaundice or cyanosis. Cardiopulmonary systems showed no abnormality. Breasts and nipples were pendulous and in normal appearance with no nodularity or retraction. There was no tenderness or organomegaly in abdomen. Ultrasonography of hepatobiliary system, spleen, pancreas, kidneys, urinary tract and bladder were normal limit. Vulvar physical examination at the time of initial presentation revealed a single, well demarcated, scaly, white ulcerated mass $2 \mathrm{~cm}$ in diameter on right labia minor. The incisional biopsy from the mass disclosed vulvar epidermal neoplasm including proliferation of tumoral squamous cells with high N/C ratio, hyperchromatic to vesicular nuclei occasionally multinucleated, conspicuous nucleoli, eosinophilic cytoplasm with distinct cell borders which confined to basement

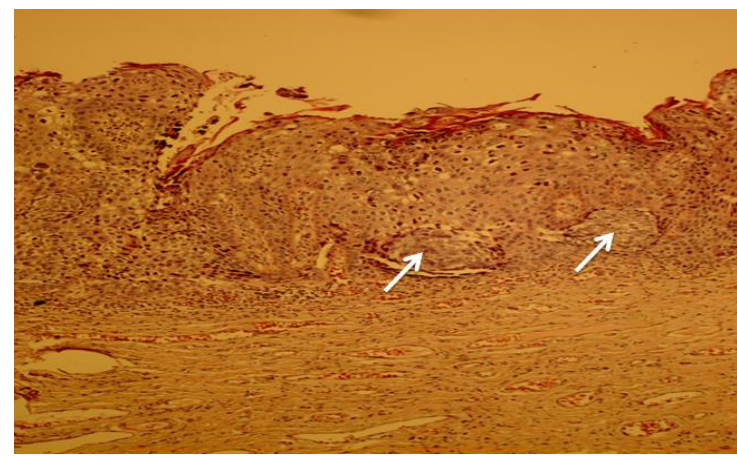

A

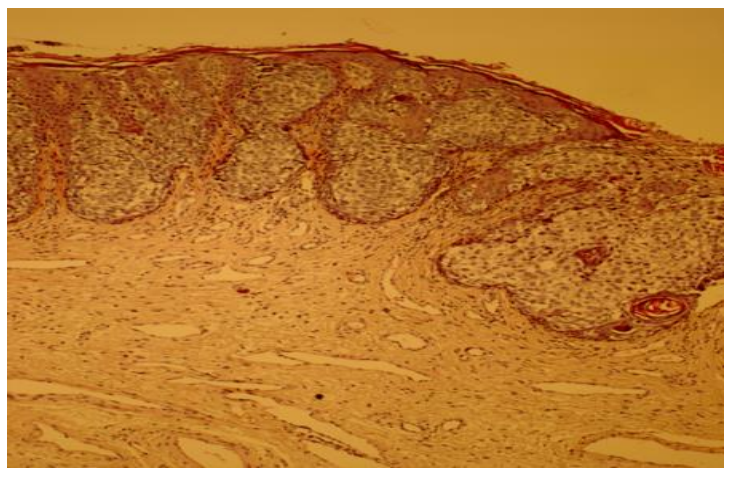

C membrane. Few dyskeratotic cells and atypical mitosis were found (Figure 1. A,B). Also there were neoplastic proliferation of atypical cells with high N/C ratio, vesicular nuclei, occasionally multi- nuclei, conspicuous nucleoli, glandular cytoplasm with distinct cell borders in epidermis in sheets and nest growth pattern (Figure 1. $\underline{A, B, C, D})$. Papillary dermis contained many melanin continence macrophages. Immunohistochemistry findings were as following: the pagetoid cells were positive for cytokeratin CK7 and focally positive for carcinoembryonic antigen (CEA) and negative for $\mathrm{S} 100$ protein, P53 and Melan A. The tumoral squamous cells were positive for cytokeratins CK5/6, P16 and p63, and negative for cytokeratin CK7 and CEA (Figure 1. E,F,G). Therefore mass lesion was diagnosed as primary Paget's disease associated with vulvar intraepithelial neoplasm (VIN) III.

Surgical treatment which were perforated for patient was radical vulvectomy with wide safe margins which including resection of clitoris, bilateral minor labia and $1 / 5$ of major labias. On pathologic examination superior, inferior and deep margins were free from tumor. Also vaginal and peripheral margins were free from tumor.

For rule out anal-rectal or urothelial malignancies colonoscopy and cystoscopy and control biopsies were performed which shown normal results. Imaging studies of abdomen and pelvis including CT scan, also revealed normal findings. The postoperative period was uneventful. The patient was disease free in the following 16 months. Participant consent was obtained before the patient was enrolled in this study.

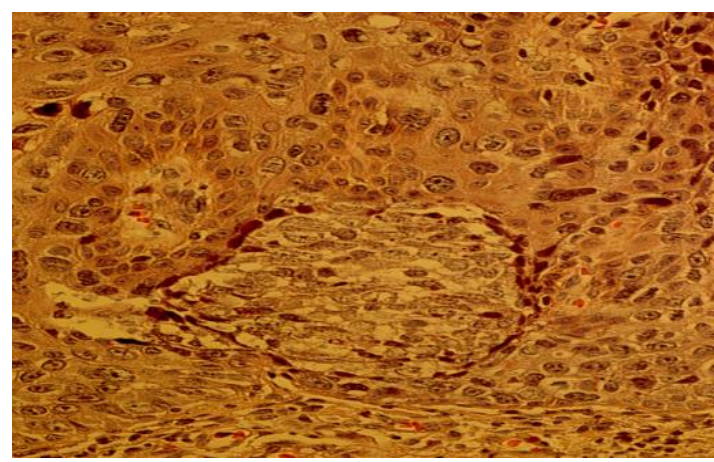

B

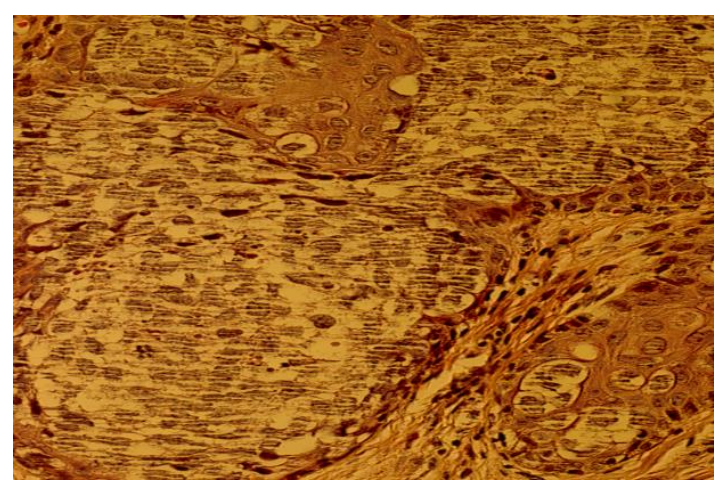

D 


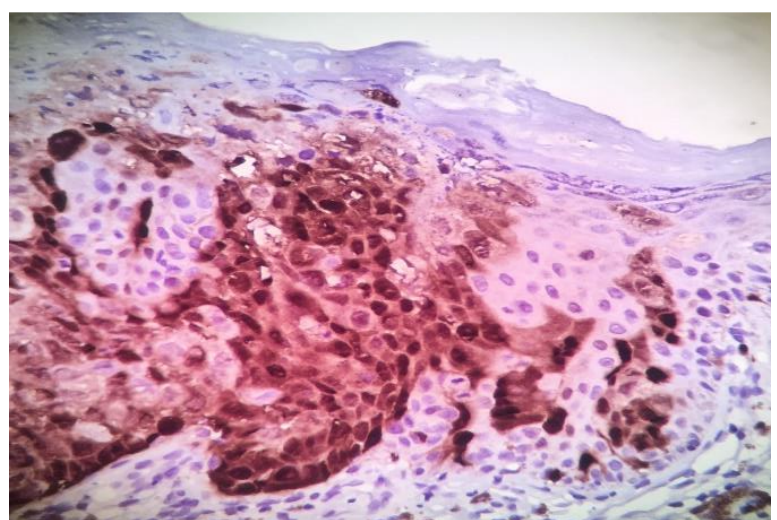

$\mathbf{E}$

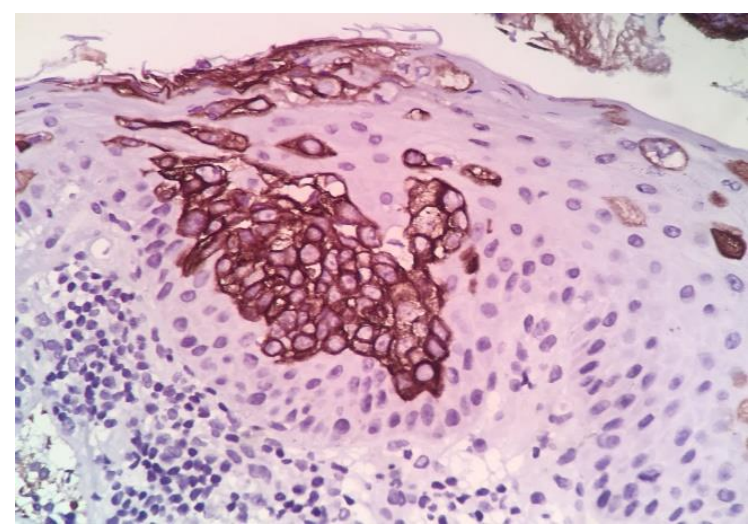

$\mathbf{F}$

Fig. 1. Histopathologic findings of incisional biopsies from the vulvar mass (A, B), Full thickness replacement of epidermis by tumoral squamous cells (VIN3) with neoplastic proliferation of glandular cells in nests pattern (Paget's disease) (arrows) (100X and 400X, respectively). (C, D) Near to totally replacement of epidermis by neoplastic proliferation of glandular cells (Paget's disease) (100X and 400X, respectively), (H\&E). E: p16 expression in VIN3, X400(IHC). F: CK7 expression in Paget's disease, X400(IHC). G: CEA expression in Paget's disease, X400(IHC).

\section{Discussion}

The vulvar Extramammary Paget's disease is a rare intraepithelial adenocarcinoma which has a peak of incidence in the fifth to eight decades of life. It manifests as a chronic eczematous, erythematous, well bordered plaque (2). It is secondarily due to pagetoid distribution of malignant cells from contiguous underlying malignancy (2). VIN is considered a rare of slow growing premalignant lesion and is mostly associated with HPV type 16 (5). Symptoms are usually long-lasting itching, burning and pain (4). As mentioned before, EMPD including vulvar Paget's disease usually manifests as a plaque, but in this case, a patient presented with a mass in vulva, is not common, but this might be due to much pruritus and reactive inflammatory changes.

The neoplastic cells of VIN3 and EMPD derivate from epidermal multipotential stem cells, therefore this fact explained concurrence of both neoplasms in same lesion (7-9).

Recently Wilkinson et al. classified vulvar Paget's disease into two types based on the source of the tumoral cells: Cutaneous (primary) Paget's disease (in situ Paget's disease, in situ with invasive Paget's disease, Paget's disease as a demonstration of an underlying cutaneous neoplasm) and noncutaneous (secondary) Paget's disease (Paget's disease as a demonstration of anal-rectal adenocarcinoma, Paget's disease associated to other adenocarcinoma, Paget's disease as a demonstration of urothelial in situ or invasive carcinoma). Each classification has three subtypes: primary including: intraepithelial cutaneous Paget's disease of the usual type, intraepithelial cutaneous Paget's disease with invasion, and intraepithelial cutaneous Paget's disease as a manifestation of underlying skin appendage adenocarcinoma. The secondary including: Paget's disease of anorectal origin, Paget's disease of urothelial origin, and Paget's disease of other origin. These three subtypes, clinical presentations can be similar but immunohistochemical studies can differentiate them. The clinical presentations of subtypes of Paget's disease may be similar, but immunohistochemical studies may be different (10).

There are some other differential diagnosis for concurrence of VIN3 and EMPD which has similar features in clinical manifestations but different in microscopy and immunohistochemistry findings, that we explain main of them as following:

Pagetoid squamous cell carcinoma in situ (SCCIS) manifests by pruritus and erythematous lesions (11). The pathologic studies reveal nests growth pattern, composed of tumoral cells with abundant, pale-staining cytoplasm in a pagetoid distribution within the epidermis (12). Immunohistochemistry (IHC) studies express CK7, CK19 and is negative for mucin and carcinoembryonic antigens (11). In EMPD with Bowenoid features, patient has a nodule and oozing erythematous painful patch (9). The histologic studies display epidermal nests of round and large tumoral cells with pale cytoplasm, and pagetoid distribution (9). IHC studies express CEA, CK7 and CAM5.2 while the stains for CK20, HMB45, S100 and P63 are completely negative (9). Lichen Sclerosis present with severe erythematous and papular lesions covered by silvery scales, with itching, pain, discoloration and changes in the vulva structure (13). The pathologic studies reveal typical hyperplasia of spinous layer and lymphocytic infiltration (CD4 \& CD8), located initially in the upper layer of dermis and subsequently also in the deeper layers (13). Melanoma manifests by nodular hyper pigmented lesions (14). The histologic studies display epidermal nests of pleomorphic tumoral melanocytes with irregular nuclei and prominent eosinophilic nucleoli with multiple atypical mitotic figures (14). IHC studies express HMB45 and S100 (14). EMPD with urothelial carcinoma origin, presented with worsening eczematous lesions and primary involvement of vulvar vestibule and periurethral mucosa (15). The pathologic studies reveal tumoral sheets of neoplastic cells with pleomorphism, nuclei 
hyperchromasia and multiple atypical mitotic activity (15). IHC studies express CK7, uroplakin 3, P16 and P53, but is negative for CK20 (15). The clinical presentation of anorectal originated EMPD, is eczematous and pruritic rash (16). IHC studies express P63, K903, CK5/6 and negative for CK20 and TTF1 (17).

But about in this case, concurrence of EMPD and VIN3 manifests as a mass in minor labia and microscopic features were high N/C ratio, vesicular nuclei, occasionally multi-nuclei, conspicuous nucleoli, glandular cytoplasm with distinct cell borders in epidermis in sheets and nest growth pattern. In immunohistochemical stains, the pagetoid cells were positive for cytokeratin CK7 and focally positive for carcinoembryonic antigen (CEA) and negative for S100 protein, P53 and Melan A. The tumoral squamous cells were positive for cytokeratins, CK5/6 and p63, and negative for cytokeratin CK7 and CEA. Our histopathologic and IHC findings were supported by another similar case report (18).

\section{Conclusion}

There are lots of diseases involving genitalia that lead to vulvar lesions. But in this rare case, we diagnosed concurrence of extramammary Paget's disease and vulvar intraepithelial neoplasia in a same lesion. Also clinical presentation as vulvar mass was unusual. So we reported it to sensitize gynecologists and pathologists for uncommon pathologies and their manifestations in vulva.

\section{Acknowledgements}

The authors would like to thank the Vice Chancellor for Research of Qazvin University of Medical Sciences and The Clinical Research Development Unit of Kosar Hospital for their collaborations during this study.

\section{Conflict of Interest}

The authors declared that there is no conflict of interest regarding the publication of this article.

\section{References}

1. Wollina U, Goldman A, Bieneck A, Abdel-Naser MB, Petersen S. Surgical Treatment for Extramammary Paget's Disease. Current treatment options in oncology. 2018;19(6):27. [DOI:10.1007/s11864-018-0545-x] [PMID]

2. Gray J, Gray A, Swan J, Eberhardt J, Mudaliar K, Tung R. Perianal extramammary Paget disease treated with topical imiquimod and oral cimetidine. Cutis. 2018;101(4):E19E22.

3. Bauman TM, Rosman IS, Sheinbein DM. Extramammary Paget's disease of the scrotum with complete response to imiquimod and photodynamic therapy. BMJ case reports. 2018;2018. [DOI:10.1136/bcr-2017-221696] [PMID]
4. Schmitt AR, Long BJ, Weaver AL, McGree ME, BakkumGamez JN, Brewer JD, et al., editors. Evidence-Based Screening Recommendations for Occult Cancers in the Setting of Newly Diagnosed Extramammary Paget Disease. Mayo Clinic Proceedings; 2018: Elsevier. [DOI:10.1016/j.mayocp.2018.02.024] [PMID]

5. Terlou A, Blok LJ, Helmerhorst TJ, Van Beurden M. Premalignant epithelial disorders of the vulva: squamous vulvar intraepithelial neoplasia, vulvar Paget's disease and melanoma in situ. Acta obstetricia et gynecologica Scandinavica. [DOI:10.3109/00016341003739575] [PMID]

6. Peterdy G, Huettner P, Rajaram V, Lind A. Trichofolliculoma of the vulva associated with vulvar intraepithelial neoplasia: report of three cases and review of the literature. International journal of gynecological pathology. 2002;21(3):224-30. [DOI:10.1097/00004347200207000-00004] [PMID]

7. Yang EJ, Quick MC, Hanamornroongruang S, Lai K, Doyle LA, McKeon FD, et al. Microanatomy of the cervical and anorectal squamocolumnar junctions: a proposed model for anatomical differences in HPV-related cancer risk. Modern Pathology. 2015;28(7):994. [DOI:10.1038/modpathol.2015.54] [PMID] [PMCID]

8. Brown HM, Wilkinson EJ. Cytology of secondary vulvar Paget's disease of urothelial origin. Acta cytologica. 2005;49(1):71-4. [DOI:10.1159/000326099] [PMID]

9. Chung J, Kim JY, Gye J, Namkoong S, Hong SP, Park BC, et al. Extramammary Paget's disease of external genitalia with bowenoid features. Annals of dermatology. 2013;25(1):88-91. [DOI:10.5021/ad.2013.25.1.88] [PMID] [PMCID]

10. Isik O, Aytac E, Brainard J, Valente MA, Abbas MA, Gorgun E. Perianal Paget's disease: three decades experience of a single institution. International journal of colorectal disease. 2016;31(1):29-34. [DOI:10.1007/s00384-015-2342-3] [PMID]

11. Armes JE, Lourie R, Bowlay G, Tabrizi S. Pagetoid squamous cell carcinoma in situ of the vulva: comparison with extramammary Paget disease and nonpagetoid squamous cell neoplasia. International Journal of Gynecological Pathology. 2008;27(1):118-24 [DOI:10.1097/pgp.0b013e318142acf0] [PMID]

12. Fujimura T, Kambayashi Y, Kakizaki A, Furudate S, Aiba S. RANKL expression is a useful marker for differentiation of pagetoid squamous cell carcinoma in situ from extramammary Paget disease. Journal of cutaneous pathology. 2016;43(9):772-5. [DOI:10.1111/cup.12743] [PMID]

13. Plagens-Rotman K, Przybylska R, Adamski Z, CzarneckaOperacz M. Skin and mucous membranes' manifestations of dermatological diseases within the genital area in females. Advances in Dermatology and Allergology/Postępy Dermatologii i Alergologii. 2018;35(2):199. [DOI:10.5114/ada.2018.75243] [PMID] [PMCID]

14. Hiratsuka J, Kamitani N, Tanaka R, Yoden E, Tokiya R, Suzuki M, et al. Boron neutron capture therapy for vulvar melanoma and genital extramammary Paget's disease with curative responses. Cancer Communications. 2018;38(1):38. [DOI:10.1186/s40880-018-0297-9] [PMID] [PMCID] 
15. Padhy RR, Nasseri-Nik N, Abbas F. Poorly differentiated high-grade urothelial carcinoma presenting as Paget's disease of the vulva with no overt urinary tract neoplasm detected. Gynecologic oncology reports. 2017;20:70-2. [DOI:10.1016/j.gore.2017.02.009] [PMID] [PMCID]

16. Wilkinson EJ, Brown HM. Vulvar Paget disease of urothelial origin: a report of three cases and a proposed classification of vulvar Paget disease. Human pathology. 2002;33(5):549-54. [DOI:10.1053/hupa.2002.124788] [PMID]
17. Pereira TC, Share SM, Magalhães AV, Silverman JF. Can we tell the site of origin of metastatic squamous cell carcinoma? An immunohistochemical tissue microarray study of 194 cases. Applied Immunohistochemistry \& Molecular Morphology. 2011;19(1):10-4. [DOI:10.1097/PAI.0b013e3181ecaf1c] [PMID]

18. Jeong Jang E, Kyung Bae Y, Hoon Shin D, Jin Lee D. Extramammary Paget's disease combined with Squamous Cell Carcinoma In Situ of the Vulva:A Case Report and Differential Diagnosis. Ann Dermatol 2016; 28(4):497498. [DOI:10.5021/ad.2016.28.4.497] [PMID] [PMCID]

\section{How to Cite This Article}

Samiee Rad, F., Farajee, S. Concurrence of Primary Cutaneous Extra Mammary Paget's Disease and Squamous Cell Carcinoma in situ of Vulva: A Case Report. Iranian Journal of Pathology, 2019; 15(1):48-51. doi: 10.30699/ijp.2019.93259.1906 